\title{
BMJ Open Task-shifted approaches to postdiagnostic dementia support: a qualitative study exploring professional views and experiences
}

\author{
Alison Wheatley (D) , ${ }^{1}$ Claire Bamford, ${ }^{1}$ Greta Brunskill, ${ }^{1}$ Karen Harrison Dening, ${ }^{2,3}$ \\ Louise Allan, ${ }^{4}$ Greta Rait, ${ }^{5}$ Louise Robinson (D) , ${ }^{1}$ The PriDem Study project team
}

To cite: Wheatley A,

Bamford C, Brunskill G, et al. Task-shifted approaches to postdiagnostic dementia support: a qualitative study exploring professional views and experiences. BMJ Open 2020;10:e040348. doi:10.1136/ bmjopen-2020-040348

- Prepublication history for this paper is available online. To view these files, please visit the journal online (http://dx.doi. org/10.1136/bmjopen-2020040348).

Received 12 May 2020 Revised 15 July 2020 Accepted 05 August 2020

Check for updates

(C) Author(s) (or their employer(s)) 2020. Re-use permitted under CC BY-NC. No commercial re-use. See rights and permissions. Published by BMJ.

${ }^{1}$ Population Health Sciences Institute, Faculty of Medical Sciences, Newcastle University, Newcastle upon Tyne, UK ${ }^{2}$ Health \& Life Sciences, School of Nursing and Midwifery, De Montfort University, Leicester, UK ${ }^{3}$ Research \& Publications, Dementia UK, London, UK ${ }^{4}$ Institute of Health Research, University of Exeter, Exeter, UK ${ }^{5}$ Research Department of Primary Care and Population Health, University College London, London, UK

Correspondence to Dr Alison Wheatley; alison.wheatley@ncl.ac.uk

\section{ABSTRACT}

Objectives To explore the views of commissioners, service development leads, service managers and senior staff in selected dementia services on increasing the role of primary care in postdiagnostic support for people with dementia.

Design Qualitative semi-structured telephone interviews and a focus group.

Setting Participants were drawn from National Health Service (NHS) Clinical Commissioning Groups, social care commissioning and a range of dementia services across primary care, secondary mental healthcare, social care and the third sector. All participants were based in England or Wales.

Participants 61 professionals, comprising 25 commissioners or service development leads; 25 service managers; and 11 team leads or senior staff.

Results Participants had varied views on whether a primary care-based approach for postdiagnostic support for people with dementia and their families was appropriate, achievable and/or desirable. Potential benefits of a task-shifted approach were continuity and a more holistic approach to care; familiarity for both patients and staff; and reduction of stigma. Key challenges included the capacity, ability and inclination of primary care to deliver postdiagnostic support for people with dementia and their families. We discovered a number of conceptual challenges to implementing a task-shifted and task-shared approach, including uncertainties around the nature of postdiagnostic support, the definition of primary care and identification of tasks that could be shifted to primary care. Conclusions Our data highlight the concerns of key professional staff around greater involvement of primary care in postdiagnostic support for dementia. Further research is needed to achieve a shared understanding and consensus over what postdiagnostic support means in the context of dementia. We will be undertaking such research in the next phase of our programme.

\section{INTRODUCTION}

Postdiagnostic dementia care and support has been defined as 'holistic, integrated continuing care in the context of declining function and increasing needs of family carers'. ${ }^{1}$ Due to our ageing populations, and
Strengths and limitations of this study

- We included the views of a range of participants in terms of their role, sector and experience of different service models.

- No comprehensive sampling frame exists for dementia services within the UK, therefore not all unique service models will have been included.

- However, our approach to identifying services was robust and included extensive desk-based research, a survey of commissioners and further snowball sampling.

- A limitation of the study is that we were unable to include professionals in Scotland and Northern Ireland.

increasing pressure on specialist services, the 2016 World Alzheimer Report recommended that postdiagnostic dementia support should move towards a 'task-shifted and task-shared healthcare model'. ${ }^{1}$ This involves shifting tasks from specialist, secondary care services to generalist healthcare settings, such as primary care, and/or sharing tasks between medical professionals and non-medical staff, for example moving tasks from general practitioners (GPs) to dementia case managers (usually nurses or social care professionals). ${ }^{12}$ A similar shift has occurred in the management of other long-term conditions, such as diabetes and depression. ${ }^{3-8}$

There has been an international, ${ }^{1}$ and in the UK national, ${ }^{9}$ policy emphasis on increasing the role of primary care in postdiagnostic dementia support. A recent systematic review of primary care-based interventions ${ }^{2}$ found that primary care case management demonstrated the greatest impact on outcomes, for people with dementia, their family carers and costs of care. ${ }^{10-13}$ However, despite evidence of effectiveness, there is limited guidance on 
how to successfully implement and sustain a primary carebased approach in practice.

The need for improved postdiagnostic support in dementia has been demonstrated by international carer surveys. ${ }^{14}$ In the UK, despite the existence of a national dementia strategy, ${ }^{15}$ around $50 \%$ of people with dementia reported receiving insufficient support after diagnosis. ${ }^{16}$ A variety of innovative postdiagnostic support projects have been piloted in the UK to address this shortfall, some of which have strong connections with primary care. ${ }^{17-21}$ The aim of this UK study was to explore the views and experiences of key professionals, including commissioners and managers of dementia services, on the concept of a task-shifted and task-shared approach.

\section{METHODS}

This study used qualitative methods to explore the views and experiences of key professionals on 'task-shifted' and 'task-shared' approaches to postdiagnostic support. All analysis was conducted according to the standard procedures of rigorous qualitative analysis ${ }^{22}$ and we drew on a range of practices from different traditions, described in more detail below. We used the Standards for Reporting Qualitative Research (see online supplemental file 1) reporting guidelines (see online supplemental file 2). ${ }^{23}$ The work reported here forms part of a larger programme to develop and evaluate new approaches to primary carebased, postdiagnostic dementia support; subsequent workstreams investigated the views of other stakeholders, including people with dementia, their family members and frontline staff.

\section{Sample and setting}

The sample comprised service commissioners and senior staff of health, social care or third sector services providing postdiagnostic dementia support across the UK; to obtain the fullest picture, we aimed to recruit a service manager and commissioner linked to each service. Since no formal sampling frame exists, services were identified primarily through extensive desk-based research, comprising internet searches and reviews of relevant documentation, such as recommendations for practice and lists of award winners. Additional services were identified through an e-survey of commissioners in England. ${ }^{24}$ The services thus identified were reviewed, with priority given to those which: stated they provided care across a full dementia care pathway; had been recognised as providing good practice (eg, through winning an award; named in national documents); offered a range of components of care; and/ or were either based in or appeared to have clear links with primary care. Sampling continued iteratively, with suggestions from both the e-survey and interview participants followed up. The concept of 'data saturation' in qualitative research has been critiqued $^{25}$; for this study, we defined 'saturation', or the data adequacy of our sample, as the point at which no further new participants or novel ways of organising postdiagnostic support had been identified.

Potential participants were initially approached via email; all email addresses were obtained from public sources (eg, service websites) or from direct enquiries to the service or commissioning group. Participants who did not respond after three contacts were excluded from the study.

\section{Data collection and analysis}

Data were collected between March and August 2019 by GB (clinical psychologist) and AW (sociologist). A topic guide for interviews/focus group was informed by existing literature ${ }^{26}$ and refined following one initial interview and focus group. Questions covered three main topics: details of current services commissioned/provided for people with dementia and their families; views on task-shifting and task-sharing postdiagnostic dementia services; and views on National Institute for Health and Care Excellence (NICE) recommendations for practice (see online supplemental file).

Interviews were conducted by telephone. This was for practical reasons: while there are potential disadvantages to telephone interviews, such as lack of body language cues, ${ }^{27}$ their use enabled us to access a highly geographically diverse sample and allowed professionals to participate with minimum disruption to their work. It was appropriate as we purposively sampled participants for their specific expertise. ${ }^{27}$ The focus group was carried out face to face. Participants were offered the option of a focus group where pre-existing groups wished to take part collectively; only one such group was identified.

All interviews and focus groups were audio recorded, transcribed (by an external company; all data were transmitted over secure connections), checked and pseudonymised for analysis (by CB, GB and AW). An inductive, thematic approach to analysis was employed. ${ }^{28}$ A coding frame was developed iteratively by CB, GB and AW through a series of data workshops throughout the period of data collection; initial transcripts were read and reread for data familiarisation, and then annotated line by line with suggested themes. Themes from all researchers were integrated and refined through group discussion, and iterations of the coding frame were tested through application to new transcripts using the constant comparative method, ${ }^{29}$ until no further new themes were generated. Data were subsequently coded by GB and AW using NVivo V.12. A small number of transcripts were coded by both researchers to ensure concordance; differences were resolved through discussion. Narrative summaries, a form of 'analytic memo', 30 of each code were written by two researchers $(\mathrm{CB}, \mathrm{GB}$, AW) independently; this process enabled us to check the coherence of each code and compare how different researchers made sense of the data. Summaries were discussed in further data workshops and integrated to produce trustworthy final versions. 


\section{Patient and public involvement}

We established a Dementia Care Community (DCC) group to inform all stages of the research, bringing together people with dementia, their family members, health and social care professionals from a range of organisations, and voluntary sector workers. ${ }^{31}$ The DCC met regularly and were involved in refining the sampling frame and topic guides, and discussing the findings.

\section{RESULTS}

Interviews were completed with staff involved in 40 of the 57 services approached. Three services advised us to approach a different service; we received no response from potential participants in 10 services; and one service did not have capacity to participate. A further three services, one in Scotland and two in Northern Ireland, were abandoned; despite a 9-month period of negotiation and numerous contacts with different people, many of whom requested duplicate documentation and raised recurrent queries, we were unable to achieve the necessary research governance permissions for these prior to the end of our data collection period. A broad range of different services providing a range of interventions were approached: these included memory services based in the secondary mental health sector; dementia practitioners based in GP practices; community dementia support teams; third sector advice and support services; Admiral Nurse-led services based in various locations; and specialist services (for example, for younger people or Black, Asian and Minority Ethnic (BAME) groups). The geographical spread of the 40 services is shown in table 1 .

We completed 49 individual or joint interviews and one focus group with 11 participants (all focus group participants were members of a social care commissioning association). A breakdown of the roles and sector of the participants is shown in table 2. Professional backgrounds of participants included GP, Old Age Psychiatrist, Occupational Therapist, Admiral Nurse, Nurse and Social Worker. Some participants had personal experience of implementing dementia services in primary care, while others spoke hypothetically. Our goal of interviewing a

Table 1 Location of included services

\begin{tabular}{ll}
\hline NHS area & Services $(\mathbf{n})$ \\
\hline East of England & 5 \\
London & 7 \\
Midlands & 3 \\
North East and Yorkshire & 6 \\
North West & 5 \\
South East & 8 \\
South West & 3 \\
Wales & 3 \\
\hline
\end{tabular}

NHS, National Health Service. manager or team lead and a commissioner linked to each service was achieved for 12 services; for the remaining services, either a manager or commissioner declined to participate.

Participants identified a range of benefits and challenges to adopting a task-shifted and task-shared approach to postdiagnostic dementia support (table 3). Additionally, we identified a lack of shared understanding of certain concepts (table 3).

\section{Benefits to a task-shifted and task-shared approach}

Most frequently expressed was the idea that shifting postdiagnostic support to primary care would allow for a more holistic approach with increased continuity, as primary care would be best placed to evaluate a patient's comorbidities alongside their dementia care.

I believe, like most long-term conditions, the majority of their care probably should fit in with primary care most appropriately [...] because it's not always clear whether a change is to do with the condition [dementia], to do with other comorbidities. Sometimes juggling the comorbidities and their treatments are quite relevant when we're looking at the cognitive impairments. (S046, GP)

Another aspect of a holistic approach was the potential to explicitly join up dementia care with care pathways for other long-term conditions, end of life care and/or frailty. Participants thought that conceptualising dementia as a long-term condition was important.

A further benefit identified by participants of shifting postdiagnostic support to primary care was the familiarity and accessibility of primary care for service users. As primary care staff may already know the person with dementia, their family and their circumstances, there was potential to build on existing rapport and reduce repetition and duplication.

I think it's absolutely fantastic for it to be based within the practice, because you really get to know your families and your patients, and I'm sure that the fact that someone with dementia has got a familiar face that they trust and feel comfortable with, that if they're becoming agitated etc. and that person goes in to try and help them out... I'm sure it's half the battle. (S025, GP practice manager)

It was also thought that locating postdiagnostic support within primary care would allow for faster and more efficient communication between professionals, either because of shared systems and data or because of physical proximity. One GP practice manager described how colocation and regular multidisciplinary team meetings (MDTs) had improved care.

We have [Ambulance Trust] working from the same building, we have [Mental Health Trust] working from the same building, we have all our social prescribing, we have an education programme going in 
Table 2 Role of participants

\begin{tabular}{lccccc} 
& & & Joint health/ & & \\
& NHS & Social care & social care & Third sector & All sectors \\
\hline Commissioners/service development leads & 14 & 7 & 3 & 1 & 25 \\
Service managers & 17 & 1 & 0 & 7 & 3 \\
Team leads/senior staff & 7 & 1 & 0 & 3 & 11 \\
All staff & 38 & 9 & 3 & 61 \\
\hline
\end{tabular}

NHS, National Health Service.

there. That goes along with [Hospital], GP services, community, Macmillan, social care and everything that's already involved. Social care come to our daily MDTs as well [...] There's a bit of joined-up work that comes from everybody having access to everybody else. I think the only way you can do that is to colocate it. (S033, GP practice manager)

Moreover, some participants suggested that primary care locations would be perceived by patients to carry less stigma in comparison with mental health services or a memory clinic.

If people see that location as inappropriate, well, they're not going to go back. It doesn't really matter how much you say, so, shifting services into GP practices is helping to remove some of that stigma. (S051, manager, specialist BAME dementia service)

Some participants shared views which were specifically related to their own experiences of a task-shared model in which a dementia specialist was employed within primary care. Reported benefits of this model included a reduction in GP workload and prevention of hospital admissions.

Table 3 Views on a task-shifted and task-shared approach to postdiagnostic dementia care

\begin{tabular}{ll}
\hline Key themes & Subthemes \\
\hline Benefits & $\begin{array}{l}\text { Improved continuity for patients } \\
\text { More holistic care } \\
\text { Familiarity of setting and personnel } \\
\text { Reduction of stigma } \\
\text { Improved access to specialist } \\
\text { services when required }\end{array}$ \\
& $\begin{array}{l}\text { Primary care workload } \\
\text { Challenges }\end{array}$ \\
& $\begin{array}{l}\text { Lack of appropriately skilled staff } \\
\text { Conceptual } \\
\text { challenges around }\end{array}$ \\
the nature of: & $\begin{array}{l}\text { Dementia } \\
\text { Postdiagnostic support } \\
\end{array}$ \\
& $\begin{array}{l}\text { Primary care } \\
\text { Care coordination }\end{array}$ \\
\hline
\end{tabular}

GPs, general practitioners.
I know that when they were piloting it, they found the workload on the GPs lifted a lot. It saves a lot of GP time. And also it does stop a lot of hospital referrals and people going to A\&E in crisis. (S031, specialist dementia practitioner)

It was also suggested that having such a specialist embedded in a service could increase staff skill and awareness of dementia.

We got an awful lot at the practice out of [Dementia Nurse Specialist] working here, which you do. If you have a specialist in ENT here, you find all the doctors start learning more about ENT. Because they've got somebody to pass ideas off. So, when you've got that specialist skill in, it helps the whole service. (S033, GP practice manager)

\section{Challenges to a task-shifted and task-shared approach}

Participants identified a number of potential barriers to implementing postdiagnostic support in primary care. Commonly mentioned were the capacity of, and appetite for change in, primary care. GPs in particular were perceived as already stretched and the feasibility of adding to their workload was questioned, while the amount of time allocated to standard GP consultations were considered too short for people with dementia.

We know it takes longer to actually have a conversation with somebody with dementia and their family, so a 10-minute appointment isn't going to cut it. So, I think it's right from a medical point of view, pulling things together, but in terms of giving the person time to have that conversation, I don't know that that is the best place. (S002, Social Care Commissioner, focus group)

The level of interest, skills and knowledge of dementia among GPs was variable and this could be another barrier to implementing postdiagnostic support in primary care.

I think that's an ongoing challenge to make sure we're reaching everybody with new updates, and new experience, and best practice and so forth. And not all GPs are interested. That's the whole thing about a general practice, general practitioner. They might not have specific special interest (S046, GP) 
Participants often drew explicit parallels with diabetes care in terms of a model to emulate. However, others pointed out the increased complexity of dementia care compared with care for diabetes.

I think the difficulty is, with dementia it's so complex and there's so many potential areas of need, as I say, with things like, "Well, do you need help with toileting? Do you need help with bathing? Do you need a sitter service overnight?" It's such a broad range of needs that a person with dementia would require. (S050, NHS commissioner)

Some professionals working in secondary care identified tasks that they thought would always require specialist input. These often involved particularly complex or challenging presentations.

I think the very specialist interventions are the areas where primary care are less able to work in that way. I have to say, we have good connections with them, and they'll phone up and say, "We've got a query about $\mathrm{X}, \mathrm{Y}$ or Z. Have you got any views?" And we can talk to them and give them some guidance. But there are people who [we] do need to go and see, so that side of that, definitely I think is the area that deservedly sits with specialist mental health at the moment. (S022, manager, Memory Assessment Service)

One of the most discussed postdiagnostic support tasks to be taken on by primary care was the Quality and Outcomes Framework annual dementia review. Implementation of the review was perceived as variable, with concerns expressed about whether and how such reviews were conducted. Some participants described considerable variation between GP practices over the completion of reviews; others felt the quality was questionable, particularly since patients and carers often seemed unaware that a review had been completed.

I don't think that activity goes on to any robust level

[...] They are not aware of the GPs reviewing them.

(S012, manager, Memory Assessment Service)

While a task-shared model in which specialist staff were based in primary care could potentially address the skills gap and provide ongoing support and training, this was not seen as sustainable. This partly reflected the large number of GP practices compared with the number of specialist staff, but also concerns over how distributed staff would be supervised and the lack of opportunities for specialists to work as a team. Physical space within primary care was also a limiting factor. Furthermore, the variation in practice size meant that rolling out interventions to small practices could be challenging due to the relatively small numbers of patients or carers who would use the service.

Obviously GP practices can be quite full so they've not always got space to enable that [carers' clinic] to happen. Some of the smaller practices, it's not really always a viable option to have a carers' clinic within a small branch or smaller practice because you don't get the flow through. (S017, NHS commissioner)

\section{Conceptual challenges}

Our data show that a lack of shared understanding of a number of key terms and concepts may contribute to difficulties in shifting or sharing postdiagnostic dementia care. In this section, we will discuss the challenges we identified around conceptualising 'dementia', 'postdiagnostic support', 'primary care' and 'care coordination'.

\section{Dementia}

There was some debate about the nature of dementia, particularly around whether it was a physical or mental health condition. Multiple professionals argued that mental health services were the wrong location for dementia care, with some suggesting that locating dementia services within primary care could help bridge the gap between mental and physical health.

I think one of the big problems we have as a system with dementia is, dementia, is it a mental health condition? Is it a physical health condition? Where does it really sit? I think actually putting it more primary care space in stops those awkward situations. (S054, manager, community dementia service)

The 'medical model' of dementia was also contested by some participants, who favoured a more social or community-focused model, though not necessarily within primary care. Examples of what this might involve included: having services based in community locations, such as dementia hubs, and run by nurses or allied health professionals; having integration between health, social care and third sector providers; making services flexible and accessible, for example using drop-in sessions.

\section{What is postdiagnostic support?}

While the World Alzheimer Report defined postdiagnostic support as 'holistic' and 'continuing, , , some participants conceived of 'postdiagnostic support' as a single meeting that occurred immediately following diagnosis or a series of follow ups that were explicitly linked to titration of medication. 'Postdiagnostic support' was seen by some participants as a discrete point on the pathway rather than an ongoing process, with a strong emphasis on the peri-diagnostic period.

They have the assessment, the diagnosis, postdiagnostic support, then they're referred back to the GP. It's at this point- Well, it could be any of that pathway that they might refer to a memory support worker, but they definitely talk about a memory support worker at post-diagnostic support. (S013, dementia service manager)

Some participants raised questions around the content, delivery and outcomes of postdiagnostic support, 
compared with diagnosis where measurements of success were more straightforward.

[what] would good post-diagnostic support look like? How could we measure it, and how can everybody demonstrate that they meet those standards? And it's up to everybody how to deliver it, but at least there's a bit more equality across the board. Everybody with suspected dementia, for example, will be seen by the memory service, or will be diagnosed within fourweeks, and those kinds of things. (S021, NHS commissioner)

\section{What is primary care?}

The WHO defines primary care as 'first-contact, accessible, continued, comprehensive and coordinated care'. ${ }^{32}$ However, when discussing the recommendation that postdiagnostic support could be task-shifted, there was some confusion around what exactly was meant by "primary care'.

It depends what you mean by primary care. So, as I said, I see primary care as the whole team. So, you know, that's looking at the local authority, looking at [Charity], plus the GP, because I think the GP can't do all of those things very well really, not just the resources and signposting to all these activities, and things like that. (S042, NHS commissioner)

It all depends if primary care is GP practice, or is wider, I don't know. Because I think practice nurses do a lot, as well. (S021, NHS commissioner)

As illustrated in previous quotations, many participants focused on GPs when considering the potential for primary care to take over postdiagnostic dementia support.

\section{What is care coordination?}

The 2018 UK NICE dementia guidelines recommended that people with dementia should have a 'single named health or social care professional who is responsible for coordinating their care'. ${ }^{26}$ With the exception of services where a dementia specialist was embedded within a GP practice to review and follow-up people with dementia, few participants were providing care coordination, although some felt that GPs took on this role. Other services were meeting the 'single named person' portion of the recommendations but did not provide coordination across health and social care. Overall, many participants identified having a named point of contact to provide continuity and familiarity as important, regardless of whether or not this included care coordination.

the Admiral Nurse or Dementia Guide could provide that link around continuity and care. I think continuity of care is crucial to support people right through their journey. (S015, Admiral Nurse)

However, the terms 'coordinating care' or 'care coordination' were problematic for some participants. Those working in secondary mental health strongly associated the term 'care coordinator' with the Care Programme Approach, a UK Department of Health strategy for supporting people in receipt of mental health services who have 'complex characteristics' such as risk of harm to themselves or others or non-physical comorbidities such as alcohol misuse. ${ }^{33}$ Participants felt that this terminology was inappropriate for dementia care.

Jargon of a care coordinator - at least in the mental health world - goes with being under a Care Programme Approach, and there's no way that everybody with dementia needs to have their care under a Care Programme Approach because that's designed for a particular threshold around peoples risks and needs. So, I don't think it's that helpful to use the term 'care coordinator'. (S016, joint health and social care commissioner)

Some participants were reluctant to use the term 'care coordination' to describe their services even when they felt they were delivering the NICE recommendation; this has implications for the wording of future guidelines around this topic. Various other terms were used by participants to describe a similar role, including care planning, key worker, guide and navigator; these terms were used inconsistently and the qualifications, roles and responsibilities vary across geographical locations.

\section{DISCUSSION AND CONCLUSIONS}

Overall, participants' views varied on whether a primary carebased, task-shifted and task-shared approach for postdiagnostic support for people with dementia and their families, was appropriate, achievable and/or desirable. Participants with experience of task-shared services, for example an Admiral Nurse based in a GP practice, were often positive about the benefits of such services for both staff and people with dementia. Some other participants had concerns about the capacity and ability of primary care to take on postdiagnostic support for dementia. However, what was understood by the term 'primary care' was not always consistent; there was an emphasis on the ability of GPs to take on additional tasks, whereas a task-shared model might be more likely to involve a team or additional specialist staff. While some participants emphasised the value of multidisciplinary working along with nurse-led and/or allied health professional-led services, they did not always envision these services fitting with primary care.

\section{Study strengths and limitations}

A key strength of the study is the broad focus on postdiagnostic support from varied providers, rather than only primary care led services. Furthermore, by including participants with and without experience of postdiagnostic services in primary care we were able to explore both perceived and actual barriers to a task-shifted approach. A further strength is the number and range of participants, achieved despite the lack of a comprehensive sampling frame of all dementia 
services within the UK. We took a robust approach to identifying services, including extensive desk-based research, a survey of commissioners and snowball sampling based on emerging information from experts. While we achieved a good geographical spread of services across England and Wales, we were unable to include services in Scotland or Northern Ireland due to research governance issues. Our study is therefore not UK-wide.

\section{Comparison with existing literature}

Our study adds to existing literature on primary care-based postdiagnostic dementia care by highlighting that a lack of clarity of some key concepts is a major challenge to developing and implementing a task-shifted and task-shared approach in real world settings. Importantly, the core issue of what comprises 'postdiagnostic support', especially in terms of its longevity to end of life, ${ }^{1}$ was not always mutually understood. Existing literature on dementia support typically focuses on the first year following diagnosis. ${ }^{34}$ A lack of shared understanding has the potential to frustrate or complicate the development and implementation of appropriate postdiagnostic support for people with dementia, whether in primary care or elsewhere.

Our participants also identified the challenge of implementing recommendations without concrete, practical guidance. If primary care is to take a greater role in postdiagnostic support, issues such as scaling and sustainability need to be addressed. These can be facilitated by examples of successful models of primary care-based postdiagnostic support and/or a shared care pathway, defined as a systematic approach for improved organisation of care processes for a well-defined patient group in a well-defined time period. ${ }^{35}$ Some countries have already developed dementia care pathways, ${ }^{36-38}$ but challenges remain regarding their implementation and uptake and the unrealistic linear management approach implied within their framework. ${ }^{39}$ The term 'integrated care pathway' was developed to incorporate multi-agency involvement care beyond healthcare ${ }^{39}$; however the newer concept of a care, or service, map, which emphasises and reflects the complexity and multiplicity of ways in which patients engage with multiple services, may be more appropriate for postdiagnostic dementia support especially for service commissioners. ${ }^{40}$

Furthermore, the majority of older people with dementia have comorbidities and physical/sensory impairments. ${ }^{41}$ Some of our participants conceptualised dementia as a longterm condition with ongoing support needs and thought that it should sit alongside, and be integrated with, other long-term condition pathways, especially frailty and end of life care. However, others felt dementia should remain under the domain of secondary mental health services. This suggests that more work is needed to negotiate a shared understanding of the nature and scope of agencies involved in providing postdiagnostic support especially in terms of overall leadership responsibility. Moreover, while parallels to the care of other long-term conditions such as diabetes were identified by several participants, a question remains about whether the nature of ongoing management for diabetes and dementia is truly similar or whether the additional complexities associated with a deteriorating neurodegenerative condition such as dementia require a different approach.

\section{CONCLUSIONS AND RECOMMENDATIONS FOR FUTURE RESEARCH}

Despite a growing policy emphasis on shifting postdiagnostic dementia support from specialist secondary services to primary and community care, our data highlight the concerns of key professional staff as to whether this is appropriate, achievable and/or desirable despite the potential advantages. To improve dementia care, research is urgently needed with key stakeholder groups and care agencies to achieve a shared understanding of postdiagnostic support in the context of dementia and also to identify successful, ongoing examples of good practice, and the factors which have contributed to their success. We will be undertaking such research in the next phase of our programme with the aim of developing a good practice intervention for primary care-based postdiagnostic dementia support and evaluate their feasibility and acceptability in routine practice.

Twitter Alison Wheatley @AlisonLWheatley, Greta Brunskill @GretaAnnB, Karen Harrison Dening @KDening, Louise Allan @drlouiseallan, Greta Rait @GretaRait1, Louise Robinson @LouiseProfessor and The PriDem Study project team @ pridemproject

Acknowledgements Additional contributions to data analysis workshops were made by Ester Bellavia and Johanne Dow. Administrative support was provided by Angela Mattison. We thank the PriDem DCC for their assistance and enthusiastic involvement throughout this project.

Collaborators The PriDem Study project team also includes Joy Adamson; Sube Banerjee; Alistair Burns; Lynne Corner; Donna Buxton; Rachael Frost; Katherine Jacques; Martin Knapp; Doug Lewins; Jill Manthorpe; Sue Tucker; Kate Walters; Jane Wilcock; and Raphael Wittenberg.

Contributors AW, CB and LR drafted the manuscript. AW and GB collected the qualitative data and analysed it along with CB. LR, LA, KHD, GR and all authors made substantial contributions to the design of the study, revised the manuscript critically for important intellectual content and approved the final manuscript.

Funding This work was supported by funding from Alzheimer's Society Centre of Excellence grant number 331.

Competing interests Professor Robinson reports grants from National Institute for Health Research Senior Investigator award, outside the submitted work.

Patient and public involvement Patients and/or the public were involved in the design, or conduct, or reporting, or dissemination plans of this research. Refer to the Methods section for further details.

\section{Patient consent for publication Not required.}

Ethics approval Ethical approval was granted by NHS Research Ethics Committee Wales 3 (reference 18/WA/0349). The majority of participants provided formal written consent via email or post, although we sought verbal consent before the interview for those who had not returned a completed consent form. Participants were allocated a unique identifier, used in all quotations.

Provenance and peer review Not commissioned; externally peer reviewed.

Data availability statement Data are available upon reasonable request. The final, cleaned, anonymised dataset will be available in a repository within 12 months. Requests to use the dataset for secondary analyses should be made to Professor Robinson (ORCID: 0000-0003-0209-2503).

Open access This is an open access article distributed in accordance with the Creative Commons Attribution Non Commercial (CC BY-NC 4.0) license, which permits others to distribute, remix, adapt, build upon this work non-commercially, and license their derivative works on different terms, provided the original work is 
properly cited, appropriate credit is given, any changes made indicated, and the use is non-commercial. See: http://creativecommons.org/licenses/by-nc/4.0/.

\section{ORCID iDs}

Alison Wheatley http://orcid.org/0000-0002-6051-0286

Louise Robinson http://orcid.org/0000-0003-0209-2503

\section{REFERENCES}

1 Alzheimer's Disease International. Improving healthcare for people with dementia. In: World Alzheimer report 2016. London, 2016.

2 Frost R, Walters K, Aw S, et al. Effectiveness of different postdiagnostic dementia care models delivered by primary care: a systematic review. Br J Gen Pract 2020;70:e434-41.

3 Atlantis E, Fahey P, Foster J. Collaborative care for comorbid depression and diabetes: a systematic review and meta-analysis. BMJ Open 2014;4:e004706.

4 Boult C, Green AF, Boult LB, et al. Successful models of comprehensive care for older adults with chronic conditions: evidence for the Institute of Medicine's "retooling for an aging America" report. J Am Geriatr Soc 2009;57:2328-37.

5 Huang Y, Wei X, Wu T, et al. Collaborative care for patients with depression and diabetes mellitus: a systematic review and metaanalysis. BMC Psychiatry 2013;13.

6 Massimi A, De Vito C, Brufola I, et al. Are community-based nurseled self-management support interventions effective in chronic patients? results of a systematic review and meta-analysis. PLoS One 2017;12:e0173617.

7 Watson LC, Amick HR, Gaynes BN, et al. Practice-Based interventions addressing concomitant depression and chronic medical conditions in the primary care setting: a systematic review and meta-analysis. J Prim Care Community Health 2013:4:294-306.

8 Frost R, Rait G, Wheatley A, et al. What works in managing complex conditions in older people in primary and community care? A stateof-the-art review. Health Soc Care Community 2020:hsc.13085.

9 Department of Health. Prime minister's challenge on dementia 2020, 2015.

10 Callahan CM, Boustani MA, Unverzagt FW, et al. Effectiveness of collaborative care for older adults with Alzheimer disease in primary care: a randomized controlled trial. JAMA 2006;295:2148-57.

11 Thyrian JR, Hertel J, Wucherer D, et al. Effectiveness and safety of dementia care management in primary care: a randomized clinical trial. JAMA Psychiatry 2017;74:996-1004.

12 Mavandadi S, Wray LO, DiFilippo S, et al. Evaluation of a TelephoneDelivered, community-based collaborative care management program for caregivers of older adults with dementia. Am J Geriatr Psychiatry 2017;25:1019-28.

13 Fortinsky RH, Delaney C, Harel O, et al. Results and lessons learned from a nurse practitioner-guided dementia care intervention for primary care patients and their family caregivers. Res Gerontol Nurs 2014;7:126-37.

14 Europe A. Carers' experiences of diagnosis in five European countries. In: Report European Carers' 2018. Luxembourg, 2018.

15 Department of Health. Living well with dementia a national strategy. London, 2009.

16 Alzheimer's Society. Dementia 2015: aiming higher to transform lives. London, 2015.

17 Kelly F, Innes A. Facilitating independence: the benefits of a postdiagnostic support project for people with dementia. Dementia 2016;15:162-80.
18 Femiola C, Tilki M. Dementia peer support: service delivery for the people, by the people. Working with Older People 2017;21:243-50.

19 Brooker D, Evans S, Evans S, et al. Evaluation of the implementation of the meeting centres support program in Italy, Poland, and the UK; exploration of the effects on people with dementia. Int $J$ Geriatr Psychiatry 2018;33:883-92

20 Aldridge Z, Burns A, Harrison Dening K. Abc model: a tiered, integrated pathway approach to peri- and post-diagnostic support for families living with dementia (innovative practice). Dementia 2019;11:147130121983808.

21 Levin KA, Lithgow S, Miller M, et al. Post-diagnostic support for dementia: What can be learned from service providers' experiences model variation and information recording? Health Education 2018;118:320-38.

22 Rapley T. Some pragmatics of qualitative data analysis. In: Silverman $\mathrm{D}$, ed. Qualitative research: issues of theory, method and practice. 3rd ed. London: SAGE, 2016: 273-90.

23 O'Brien BC, Harris IB, Beckman TJ, et al. Standards for reporting qualitative research: a synthesis of recommendations. Acad Med 2014;89:1245-51.

24 Frost R, Walters K, Wilcock J, et al. Mapping post-diagnostic dementia care in England: an e-survey. J Integr Care 2020

25 Braun V, Clarke V. To saturate or not to saturate? Questioning data saturation as a useful concept for thematic analysis and sample-size rationales. Qual Res Sport Exerc Health 2019;2:1-16.

26 National Institute for Health and Care Excellence. Dementia: assessment management and support for people living with dementia and their carers NG97. 20, 2018.

27 Block ES, Erskine L. Interviewing by telephone: specific considerations, opportunities, and challenges. Int J Qual Methods 2012;11:428-45

28 Braun V, Clarke V. Using thematic analysis in psychology. Qual Res Psychol 2006;3:77-101.

29 Glaser BG. The constant comparative method of qualitative analysis. Soc Probl 1965;12:436-45.

30 Charmaz K. Constructing grounded theory. London [United Kingdom]; Thousand Oaks, California: SAGE, 2014.

31 PriDem study project team. DCC, 2020. Available: https://research. ncl.ac.uk/pridem/engagement/dcc/

32 World Health Organisation. Primary health care: main terminology, 2020. Available: http://www.euro.who.int/en/health-topics/Healthsystems/primary-health-care/main-terminology

33 Department of health. Refocusing the care programme approach. London, UK, 2008.

34 England NHS. The well pathway for dementia, 2016

35 Schrijvers G, Hoorn Avan, Huiskes N. The care pathway concept: concepts and theories: an introduction. Int J Integr Care 2012;12.

36 National collaborating centre for mental health. The dementia care pathway: full implementation guidance. London, 2018.

37 NHMRC Partnership Centre. Dealing with cognitive and related functional decline in older people. In: Clinical practice guidelines and principles of care for people with dementia, 2016.

38 National Institute for Health and Care Excellence. Dementia overview. 31, 2020.

39 Samsi K, Manthorpe J. Care pathways for dementia: current perspectives. Clin Interv Aging 2014;9:2055-63.

40 Checkland K, Hammond JON, Allen P, et al. Road to Nowhere? A Critical Consideration of the Use of the Metaphor 'Care Pathway' in Health Services Planning, Organisation and Delivery. J Soc Policy 2020;49:405-24.

41 Bunn F, Burn A-M, Goodman C, et al. Comorbidity and dementia: a scoping review of the literature. BMC Med 2014;12:192. 